\title{
Enfoque psicosocial de la diabetes. Una mirada cualitativa
}

\author{
Psychosocial approach to diabetes. A qualitative look \\ Betsy Flores-Atilano,* Dulce María Guillén-Cadena, ${ }^{*}$ \\ María Guadalupe López-Sandoval, ${ }^{\S}$ Karen Yael Alcántara-Ruíz"
}

\begin{abstract}
RESUMEN
Introducción: Un incremento en la incidencia de enfermedades no transmisibles (ENT) se ha registrado en las últimas dos décadas, la Organización Mundial de la Salud sostiene que las ENT son responsables de más de 40 millones de muertes al año, lo que equivale al 70\% de fallecimientos que se producen en el mundo, 75\% de las muertes asociadas a las mismas ocurrieron en países de medianos y bajos ingresos, lo que refleja la gravedad del problema en países como el nuestro. Material y métodos: Investigación cualitativa con diseño fenomenológico interpretativo; la recolección de datos se realizó a través de la entrevista en profundidad, observación no participante y notas de campo; como instrumento se utilizó una guía de preguntas, participaron mujeres entre 40 y 60 años de edad. Los principios bioéticos fueron los propuestos por Emanuel, el cual garantiza el reconocimiento de individualidad y anonimato de los informantes, el rigor científico de este estudio se basó en los criterios propuestos por Castillo (2003): credibilidad, auditabilidad y transferibilidad, el análisis de la información se llevó a cabo de manera secuencial mediante los cuatro pasos propuestos por Richard Krueger: 1) la obtención de información, 2) captura, organización y manejo, 3) codificación y 4) verificación participante. Resultados: Emociones, baja autoestima, falta de motivación, apoyo familiar, medio laboral y relaciones interpersonales son algunos de los temas encontrados en esta investigación. Conclusiones: Los aspectos psicosociales se encuentran íntimamente relacionados con el proceso de salud y bienestar, determinante en la vida del individuo. Esto desde el propio diagnóstico de diabetes, el cual implica un forzoso cambio conductual y apego terapéutico de cara a las diversas complicaciones potenciales en las distintas esferas del paciente.
\end{abstract}

Palabras clave: Análisis cualitativo, diabetes mellitus, vivencias, pacientes, psicosocial.

\begin{abstract}
Introduction: An increase in the incidence of non-communicable diseases has been recorded in the last two decades, the World Health Organization maintains that NCDs are responsible for more than 40 million deaths per year, equivalent to $70 \%$ of deaths in the world, $75 \%$ of the deaths associated with them occurred in medium and low income countries, reflecting the seriousness of the problem in countries like ours. Material and methods: Qualitative research with interpretive phenomenological design; data collection was carried out through in-depth interview, non-participant observation and field notes; as a tool, a question guide was used, women between 40 and 60 years old participated. The bioethical principles were those proposed by Emanuel, which guarantees the recognition of the individuality and anonymity of the informants, the scientific rigor of this study was based on the criteria proposed by Castillo (2003): credibility, auditability and transferability, the analysis of the information was carried out sequentially through the four steps proposed by Richard Krueger: 1) obtaining information, 2) capture, organization and management, 3) coding and 4) participant verification. Results: Emotions, low self-esteem, lack of motivation, family support, work environment and interpersonal relationships are some of the topics found in this research. Conclusions: The psychosocial aspects are closely related to the health and wellbeing process, a determinant in the
\end{abstract}

*Profesora de asignatura «A».

‡ Profesora titular «C», Tiempo completo.

$\S$ Profesora asociada «B». " Estudiante de Maestría en Enfermería.

Facultad de Estudios Superiores Iztacala, UNAM.

Correspondencia: BFA atilann@unam.mx, atilann@gmail.com Conflicto de intereses: Los autores declaran que no tienen.

Citar como: Flores-Atilano B, Guillén-Cadena DM, López-Sandoval MG, Alcántra-Ruíz KY. Enfoque psicosocial de la diabetes. Una mirada cualitativa. Rev CONAMED. 2020;

25(2): 75-83.

doi: $10.35366 / 94390$

Financiamiento: Ninguno.

Recibido: 27/04/2020 Aceptado: 25/06/2020 
life of the individual. This from the diagnosis of diabetes itself, which implies a forced behavioral change and therapeutic attachment for the various potential complications in the different areas of the patient.

Keywords: Qualitative analysis, diabetes mellitus, experiences, patients, psychosocial.

\section{INTRODUCCIÓN}

En la actualidad, las enfermedades no transmisibles (ENT) o crónico-degenerativas representan una de las principales preocupaciones de la población mundial, dadas sus implicaciones personales, familiares y sociales, así como su lento y progresivo aumento en las tasas epidemiológicas. La Organización Mundial de la Salud (OMS) sostiene que las ENT son responsables de más de 40 millones de muertes al año, lo que equivale al 70\% de fallecimientos que se producen en el mundo. A su vez, la diabetes está dentro de las cuatro principales ENT responsables de más de $80 \%$ de todas las muertes prematuras, misma que causa 1.6 millones de muertes al año a nivel mundial.?

En realidad no se cuenta con estimaciones mundiales sobre prevalencia de diabetes que separen el tipo 1 del tipo 2, lo anterior debido a que se requieren pruebas sofisticadas de laboratorio para distinguirlas de acuerdo con el informe mundial de la OMS.' El tipo 1 requiere dosis de insulina para la supervivencia del paciente, mientras que el tipo 2 supone una deficiente utilización de la insulina que produce el organismo de la persona que la padece. Sin embargo, lo que sí se reporta es que la mayoría de los pacientes tiene diabetes de tipo 2, misma que solía ser exclusiva de la población adulta pero que hoy en día también se da en la población infantil. ${ }^{2}$

Independientemente del tipo de diabetes que se padezca, las complicaciones en el organismo y el riesgo general de mortalidad prematura se ven incrementadas. Dentro de las posibles complicaciones a la salud derivadas de padecer diabetes se encuentran: accidentes cerebrovasculares, ataques cardiacos, daños neurológicos, insuficiencia renal, amputación de miembros pélvicos, pérdida de visión, entre otros. A su vez, en el caso de la diabetes gestacional, se requiere un estricto y adecuado control de la paciente grávida, de lo contrario aumenta el riesgo de muerte fetal y otras complicaciones asociadas. ${ }^{2}$
Son indiscutibles las complicaciones orgánicas que la diabetes conlleva para la persona que la padece. No obstante, las complicaciones se extienden en otros aspectos, como es el caso de lo psicológico y lo social. ${ }^{2}$

En cuanto a las repercusiones psicológicas que presentan las personas que padecen alguna ENT, existe una relación bidireccional entre la experimentación emocional y las enfermedades crónicas. En el caso específico de la diabetes, en general se establece que ésta puede predisponer a los pacientes a experimentar una serie de emociones negativas, tales como ira, rabia y tristeza; sin embargo, también se sabe que existen otros aspectos psicológicos como la relación entre el afecto, las respuestas emocionales y la probabilidad del desarrollo de un cuadro diabético en personas en riesgo, ya sea por herencia o aspectos conductuales como sedentarismo y mala alimentación. ${ }^{3}$

Los aspectos conductuales no son otra cosa más que el resultado del comportamiento y del estilo de vida del paciente, convirtiéndose así en su principal enemigo a vencer. Lo anterior aunado a su contexto sociocultural representa un gran reto que la persona que padece diabetes tendrá a partir de su diagnóstico. Situación que deberá modificar y que sin duda es de interés para los profesionales de la salud, ya que le proporcionarán alternativas para la orientación hacia la adopción de conductas favorables que resulten en una buena calidad de vida.

En este sentido es oportuno manifestar que la calidad de vida hace referencia al bienestar físico, social, psicológico y espiritual del individuo. ${ }^{4}$ En cuanto al bienestar psicológico, este implica satisfacción con la vida, presencia de estado de ánimo positivo y ausencia de estado de ánimo negativo (que en conjunto determinan la felicidad); y a su vez, el valor asignado a las metas, la realización del verdadero potencial individual, el alcance de logros y la satisfacción personal. ${ }^{5}$

La diabetes implica un control metabólico mediante apego terapéutico, no sólo en términos 
farmacológicos, sino también en otras medidas coadyuvantes relacionadas con el autocuidado a través de la modificación de aspectos conductuales que requieren indiscutiblemente de trabajo interno por parte de quienes la padecen.

En cuanto al aspecto social, es necesario comenzar haciendo mención sobre la imperante necesidad de un proceso de aceptación y capacitación adecuada enfocada en el autocuidado diario para lograr un control metabólico deseado por parte de los pacientes. Lo anterior, sin duda, los hará menos vulnerables de sufrir los efectos adversos de la enfermedad sobre su salud y su calidad de vida4 Esto debido a que existen estudios que reafirman la importancia en la implementación de estrategias de intervención dirigidas al mejoramiento de la respuesta adaptativa a la diabetes, puesto que un gran número de pacientes limitan sus fuentes de información a sus redes sociales más próximas y «confiables» a su entender, tales como su familia, compañeros de trabajo y amistades. ${ }^{6}$

No debe olvidarse que al hablar de la persona que padece diabetes se hace referencia a un ser humano que es evidentemente social, lo cual le confiere existencia, expresión y proyección en diversos escenarios donde se ve inmerso. Escenarios que pueden tanto sumarle o restarle en cuestión de afirmación y desenvolvimiento de su rol social, por medio del cual desempeña funciones y actividades que lo integran en la sociedad, ya sea en su familia, trabajo o comunidad. Es precisamente esta individualidad la que le permite trascendencia y mantenimiento de sus relaciones interpersonales, las cuales son indispensables para la reconstrucción continua de su imagen personal y social.7

A su vez, es oportuno mencionar otras repercusiones que conlleva la diabetes en el ámbito social, más allá de los círculos cercanos del individuo padeciente, puesto que si bien son innegables las pérdidas monetarias tanto para el paciente como su familia, también son cuantiosas para los propios sistemas de salud que impactan en la economía nacional por gastos médicos directos e indirectos. ${ }^{2}$

\section{MATERIAL Y MÉTODOS}

La investigación se llevó a cabo en el municipio de Tlalnepantla de Baz, Estado de México, en el periodo comprendido entre enero y octubre de
2019, mediante la metodología cualitativa con diseño fenomenológico interpretativo. La recolección de datos se realizó a través de la entrevista en profundidad, observación no participante y notas de campo; como instrumento se utilizó una guía de preguntas elaborada por el equipo de investigación, a partir de la revisión literaria enfocada en los aspectos psicosociales de la diabetes, misma que fue avalada por un experto.

Participaron cuatro mujeres, las cuales fueron seleccionadas de acuerdo al típico ideal según Rodríguez y Gil y cuyas características fueron las siguientes: trabajadoras de la Facultad de Estudios Superiores Iztacala de la Universidad Nacional Autónoma de México, que han vivido el fenómeno desde hace cinco años como mínimo, entre 40 y 60 años de edad, interesados en participar en el estudio, dispuestos a leer y firmar el consentimiento informado y con disponibilidad manifiesta para una posible entrevista subsecuente por cuestiones de verificación participante para mantener el rigor metodológico. ${ }^{8}$ El total de participantes se definió a partir de saturación teórica, tal y como refiere Morse, al mencionar que para alcanzar la saturación se requiere de la conjunción de diversos factores como la calidad de los datos, el alcance del estudio, la naturaleza del tema, la información útil obtenida de cada participante, así como el método y el diseño de estudio cualitativo utilizado, es decir, que en realidad no se busca una representación estadística, sino una interpretación adecuada del discurso sobre un objeto de estudio determinado. ${ }^{9,10}$ Las entrevistas se llevaron a cabo dentro de su espacio laboral, teniendo una duración entre 40 y 60 minutos.

El análisis de la información se llevó a cabo de manera secuencial mediante los cuatro pasos propuestos por Richard Krueger, mediante: 1) la obtención de información, 2) captura, organización y manejo, 3) codificación y 4) verificación participante. Lo cual significa que la información obtenida de cada entrevista fue transcrita y posteriormente leída para la identificación de códigos y tópicos de trabajo para el análisis. Después, se recuperaron segmentos codificados para contrastar los temas derivados del análisis a partir de los discursos de los participantes, corroborando y verificando su esencia con los mismos. Finalmente, se identificaron diferencias y semejanzas en los discursos para 
establecer patrones de significación y pertinencia por temas. $^{8}$

En cuanto a los principios bioéticos durante la realización del estudio, el equipo de investigación se basó en el modelo propuesto por Emanuel, el cual garantiza el reconocimiento de individualidad y anonimato de los informantes, manteniendo integridad en su ideología, reservando su identidad y respetando sus elementos culturales, juicios y opiniones con absoluta discreción. A su vez, dicho modelo sustenta sus principios en la normatividad de la investigación bioética reflejada en la Declaración de Helsinki, el Código de Núremberg, el informe Belmont y las normas del Consejo para la Organización Internacional de Ciencias Médicas." De manera simultánea, se tomó como referencia el artículo 100 del Título Quinto de la Ley General de Salud en materia de investigación para la salud, especialmente en lo que se refiere a su posible contribución a la solución de problemas de salud.12

Para mantener el rigor científico de este estudio cualitativo se adoptaron los criterios propuestos por Castillo (2003): credibilidad, auditabilidad y transferibilidad, mismos que se refieren respectivamente al reconocimiento de los hallazgos como reales tanto por los participantes como para los que han experimentado el fenómeno, a la neutralidad del análisis e interpretación de la información mediante la confirmación de otros estudios con posibles hallazgos similares y la posibilidad de transferir los hallazgos a otros contextos en estudios posteriores. 13,14

Se empleó el paradigma cualitativo para la realización de este estudio por su enfoque subjetivo, debido a que se adentra en las experiencias humanas, a partir de su esencia epistemológica que radica en la propia experiencia del sujeto y no en lo cuantificable o medible. En lo referente a la aproximación fenomenológica, se debe considerar que ésta no parte de presuposiciones, el fenómeno de estudio se coloca antes de cualquier creencia y de todo juicio.

El diseño fenomenológico interpretativo propuesto por Martin Heidegger fue elegido para la realización de este estudio debido a que muestra una relación del ser humano con su mundo, de donde surge el término Dasein que significa serahí, es decir, un ser humano en el mundo que es abordado por sí mismo en cada experiencia de vida. Experiencia que al ser traída por él mismo lo hace consciente de su propia existencia y puede ser expresada a través del lenguaje. ${ }^{15}$

\section{RESULTADOS}

Después de haber realizado un profundo análisis de las entrevistas de acuerdo con los criterios de Krueger, y bajo estricto apego al rigor de Castillo (ambos mencionados en el marco metodológico), se encontraron los siguientes temas:

\section{Temas}

\section{El lado psicológico de las emociones}

De acuerdo con Chóliz (2005) en su trabajo titulado «Psicología de la emoción: El proceso emocional», se puede definir a la emoción como una reacción subjetiva al ambiente, la cual viene acompañada de cambios fisiológicos y endocrinos de origen innato, mismos que son influidos por la experiencia. La función de la emoción resulta importante para el organismo humano, puesto que funge como coadyuvante adaptativo a lo que nos rodea. ${ }^{16}$

Es a partir de lo anterior que se exponen algunos fragmentos de la información proporcionada por las personas que participaron en el estudio.

\section{En voz de las participantes:}

Rosa: «Pues al principio no lo asimilaba. Ya después, pues... como que me siento culpable. Culpable porque me digo yo misma: tengo la culpa de que me haya dado diabetes por llevar una vida sedentaria y por no llevar una buena dieta...».

Algunos pacientes reaccionan ante el diagnóstico de diabetes con negación, ira, culpa y depresión antes de aceptar el reto que implica el tratamiento. ${ }^{10}$ Éstas han sido las emociones que más se presentan en este tipo de pacientes, principalmente cuando reciben el diagnóstico.

Gabriela: "Estar bien es levantarme con muchas ganas. Venirme a trabajar, estar contenta conmigo misma. No siento así como que me duele acá, me duele allá..., no pienso en eso. Y bueno... no me duele nada tampoco. jVerdad! -risas-».

Según Chóliz, la experiencia de una emoción involucra un conjunto de cogniciones, actitudes y 
creencias sobre el mundo, para valorar una situación concreta y, por tanto, esto influye en el modo en el que se percibe dicha situación. Es decir, el impacto es de mayor amplitud y trascendencia, dada la cuestión emotiva. ${ }^{16}$

De acuerdo con Heidegger, la manifestación de las respuestas humanas es «existencia». Proceso que no sólo hace referencia al hecho de estar presente en el mundo, sino de experimentar y estar inmerso en el fenómeno, lo cual le permite sentirlo vívido y es ahí cuando el ser se manifiesta y por ende, existe. ${ }^{15}$

\section{La baja autoestima y la falta de motivación}

Según Páez, el contexto sociocultural es una fuente de autoestima. Se puede definir como la estima que se tiene de sí mismo a partir del esfuerzo personal y logros alcanzados dentro de la sociedad para tener respeto y reconocimiento de los demás. ${ }^{17}$

A su vez, es oportuno mencionar que la motivación es aquel impulso o necesidad que hace que una persona realice determinada acción para lograr una meta para el bienestar, esta puede manifestarse de dos formas: interna o externa. De acuerdo con Castillo (2017), la motivación y los conocimientos sobre la dieta son aquellos factores personales principales que influyen en la adherencia terapéutica de un paciente. ${ }^{18,19}$ A continuación se presentan algunos fragmentos ilustrativos de discurso de las participantes:

Rosa: "Me veo en el espejo y digo: estoy refea, refea, vieja y ya diabética».

Sandra: «Saber que ya estaba diabética...no sé pero me cambió mi carácter, me hice muy muy cerrada, muy en mi casa, como que mi salvación era mi casa y estar ahí, yo sentirme bien sólo en mi casa».

Rosa: "O sea y luego ya de repente digo: ¡No! ¡Sí tengo que echarle ganas, quiero hacer esto, quiero hacer lo otro!».

Gabriela: "No me presiono así como decir: ¡Ah! Se me antoja esto, pero no lo puedo comer. Hay veces que lo pruebo, hay veces que no se me antoja... y me siento bien, me siento bien porque no me privo, pero tampoco exagero a comer cosas que sé que me hacen daño».
Sandra: "Te digo, nos dio una información tanto alimenticia que supe qué sí puedo comer y qué no, como las verduras...en cambio ahora puedo hacer una ensalada y combinarla con otras legumbres y sí las como, ya es diferente y gracias a eso ya busco».

Rosa: "No me siento motivada».

Sandra: "Una dieta que la dieta no ¿verdad? No la puedo llevar. Es fecha que me cuesta mucho trabajo porque no tengo buenos hábitos alimenticios... este... no me gustan las verduras. ¡No me gustan! Y me encantan los dulces... me encantan. ¡Los chocolates me fascinan!». "Cuando empecé, que me detectaron, yo tenía 19 años... me dice el doctor que la diabetes es una enfermedad que era muy difícily más en aquellos tiempos que nos decían que no había tantos medicamentos y que, así me lo dijo él: ¡Usted se va a morir!».

Al padecer una ENT, la autoestima del paciente se ve afectada por diversas razones, como el deterioro físico, el cambio del estilo de vida, el tratamiento y el proceso de aceptación de la enfermedad que en general es bastante largo. Lo cual coincide con Ledón, quien refiere que tienden a aislarse, puesto que existen cambios en la personalidad y el carácter en el periodo de ajuste mientras llegan a la aceptación. ${ }^{5}$ Cuestión que puede entorpecer la adherencia terapéutica de la persona que padece diabetes.

Asimismo, esto coincide con Castillo (2017), quien afirma que los factores que condicionan la adherencia deficitaria están relacionados con la falta de conocimientos sobre la dieta a seguir y la ausencia de motivación por realizar adecuadamente el tratamiento. ${ }^{19}$

Desde esta perspectiva, la fenomenología desde el punto de vista de Heidegger busca la comprensión y la interpretación de cada caso particular de la experiencia, en el modo en que el vivir se tiene a sí mismo manifestado en el ser y el modo en que ese vivir habla de sí mismo.15

\section{El lado social}

El ser humano es un ente social con interacciones dentro de su medio ambiente en grupos sociales de características determinadas como ideología, religión, estatus económico e incluso de salud. A su vez, este ente asume un rol social que de manera 
reflexiva le brinda la aprobación o negación a través de sus relaciones interpersonales, laborales, sentimentales, familiares, etcétera, mismas que son parte fundamental de su desarrollo..$^{15}$

Este tercer tema se divide en tres subtemas: a) apoyo familiar y b) medio laboral, que abordan los dos contextos más mencionados por las participantes; mientras que el último: c) relaciones interpersonales: ¿reto o alivio?, incluye algunos ejemplos de las experiencias más difíciles de convivir socialmente y la importancia que tienen las redes de apoyo de las pacientes para afrontar aquellos obstáculos sociales derivados de su padecimiento.

\section{a) Apoyo familiar}

La familia es reconocida como la principal red de apoyo de los pacientes para llevar a cabo las indicaciones médicas, tales como el cambio de alimentación, el ejercicio y el tratamiento, además de fungir como protector y aporte económico. De esta manera, se expone la importancia que tiene el núcleo familiar para que el paciente afronte su nuevo estilo de vida y haga una reflexión de lo positivo y lo negativo respecto a su enfermedad.

\section{En voz de las entrevistadas:}

Rosa: «Y mi familia, pues... no, pues mi familia me apoya, ¿no? Me dicen: si se cuida sí va a durar. Sí va si se cuida. Si puede llevar una vida... vida normal».

Gabriela: «Mis hermanas. Mucho de mis hermanas. Es el apoyo de: "cuídate, no comas esto». O cuando voy a... o tenemos reunión, tampoco ponen cosas así que se te antojen y no lo puedas comer. Tengo una hermana que... ella nos pone, por ejemplo, una ensalada de pepinos, jícama... cosas así. Y una manzana o una papaya, fruta... No nos pone cosas así como empanizados, fritos... Cosas saludables. jY es para todos!».

Al abordar la experiencia social al padecer diabetes, las participantes obtuvieron apoyo del primer grupo social: la familia y, por supuesto, de las amistades. El apoyo proporcionado fue positivo y consistió en actos solidarios al comer lo mismo como muestra de empatía, e incluso también en cuanto a los alimentos que no podían consumir frente a los pacientes. Además de prestarles atención en cuanto a su apego terapéutico, citas con el médico y, desde luego, aspectos referentes a la promoción del autocuidado.

Coincidiendo con Ávalos (2017), quien indica que lograr un buen control de la diabetes no sólo depende del paciente o de los profesionales de la salud; la familia también es un recurso indispensable a incluir dentro de las estrategias e intervenciones de salud, debido a que ésta puede favorecer una buena evolución de la enfermedad o un desenlace inesperado. ${ }^{20}$

Todo lo anterior influye en el paciente para la aceptación de su enfermedad y adopción de estilos de vida saludables, razón por la cual Guerrero (2016) considera que es necesario señalar que de no diseñar acciones personales y conjuntas que involucren a la familia y a los profesionales de la salud, el intento del paciente por llevar un adecuado manejo de la diabetes se convertirá en el principal factor de riesgo para experimentar una pobre calidad de vida. ${ }^{21}$

\section{b) Medio laboral}

Una parte importante del entorno social del paciente con diabetes es su medio laboral, puesto que se trata de un lugar donde se ha desarrollado gran parte del tiempo, poniendo en práctica sus habilidades para generar un ingreso económico para sostenerse a sí mismo y a su familia. Se trata de un entorno social donde desempeña un rol y mantiene un cierto estatus y con ello relaciones profesionales y personales que pueden derivar en amistades que pueden significarle redes de apoyo ante su enfermedad.

\section{En voz de las entrevistadas:}

Gabriela: «En el trabajo no. No tengo problemas. Así muy tranquila, ¿no? No tengo. Así de compañeros o de jefes. Me mantengo ocupada, no pienso en nada, no me enojo con nadie. Lo contrario, me estoy riendo con ellos, porque cada vez cualquier cosa que se les ocurre... estamos riendo».

Sandra: «Somos un grupo en el cual, ya sabe... pues ya estamos grandes. Unas compañeras y yo somos diabéticas, pero nos ayudan los compañeros. Ellos se llevan la basura. Que si vamos a cargar pesado: «iNo, no. Ustedes no!». Ellos cargan. 
Ya convivimos más. Mi ambiente es diferente. Ellos nos andan ayudando y todo eso. Y la verdad, gracias también al jefe que también nos ayuda y nos apoya».

El trabajar y padecer diabetes impacta en la manera que tienen los pacientes para sobrellevar su enfermedad mediante el apoyo recibido de parte de sus compañeros e incluso de sus superiores al encontrar comprensión en ellos. Lo cual genera una atmósfera laboral armónica que permite desarrollar con normalidad el trabajo diario.

Coincidiendo con Martínez, quien afirma que no sólo la familia y la pareja, sino también los compañeros del entorno laboral y escolar, y las relaciones sociales en general, son fuentes importantes de apoyo para las personas que padecen diabetes, puesto que representan un psicosocial de adaptación a la enfermedad y el mantenimiento de la salud.22

A propósito de lo anterior, Salazar refiere que una organización laboral es un microambiente compuesto por individuos, puestos y áreas para desempeñar funciones que conllevan actividades específicas que permiten el desarrollo del individuo. Implica aspectos sociales como el compañerismo, conflictos interpersonales, etcétera, lo cual genera aptitudes y actitudes particulares que traen motivación y expectativas en las personas. ${ }^{23}$

\section{c) Relaciones interpersonales: ¿reto o alivio?}

Las relaciones interpersonales son aquellos vínculos que se crean a partir de la interacción con otras personas, éstas pueden ser amigos, vecinos, familia, compañeros de trabajo, etcétera, y que se basan en la demostración de emociones y sentimientos, intereses como el arte y el cine, y el intercambio de pensamiento que facilitan el desarrollo personal y social de la persona con su contexto social, cabe señalar que el componente esencial de esa interacción es la comunicación.

\section{En voz de las entrevistadas:}

Sandra: «Sífue muy difícil porque pues... estaba en esa edad. Yo estaba en la universidad. Pues empezaba a salir con amigos, a tener... Buscar novio, pues no. Yo no. Preferí, entonces, no hacerlo. Definitivamente dejé ese tipo de actividades: de tener amigos, de dedicarme más a estar en casa. Entonces yo ya no salía. No, jeh! Ya no más en la escuela. No terminé mi escuela y empecé a estar enferma».

La dificultad en las relaciones interpersonales de las participantes es derivada de la convivencia con determinadas personas que con sus actitudes y acciones perjudican el sentir del paciente con diabetes, provocándole aislamiento del medio social y con ello las acciones de autocuidado, lo que afecta su estado de salud. A su vez, al saberse con diabetes, existe un conflicto interno para poder relacionarse con su medio social.

Las amistades son una extensión de la vida social de la persona, son relaciones interpersonales donde el vínculo es fuerte y duradero como el de la familia, así su importancia para superar obstáculos de la vida y, por supuesto, de salud como es el caso del paciente con diabetes.

El apoyo por parte de las amistades del paciente puede influir de una manera positiva o negativa en el pensamiento y comportamiento ante su situación actual de salud, promoviendo así el autocuidado o llevar a un estado de ánimo desafortunado para el afrontamiento de la enfermedad.

Rosa: «Me siento feliz también cuando platico con algunas personas que son positivas y todo se me olvida. Que soy diabética, o sea... me siento bien». "Pues, bueno... algunas amistades si me dijeron: ¡No, diabetes es muerte! No vas a durar mucho...».

Cuando se presentan amistades con un apoyo negativo a partir de comentarios u opiniones hacia alguien que padece diabetes suelen tener un resultado negativo, lo cual deja al paciente sin esperanzas.

El apoyo de las amistades se visualiza de dos maneras: las palabras motivacionales hacia el cuidado que debe tener de ahora en adelante con la enfermedad y, por otro lado, los comentarios negativos influenciados por las creencias que se tienen socialmente sobre la enfermedad, es decir, la diabetes como sinónimo de muerte o amputación, lo cual provoca miedo y ansiedad ante el futuro inmediato.

La investigación psicológica y social ha puesto de manifiesto la importancia de las relaciones interpersonales como fuente de recursos esenciales 
para la promoción de la salud y bienestar de los pacientes a través de la movilización e intercambio de diversas modalidades de apoyo social. ${ }^{24}$ Esta relación se ha constatado, más concretamente, en el caso de las personas con enfermedades crónicas. ${ }^{25}$ Estos pacientes necesitan sentirse apoyados por las personas que les rodean (sus familiares, compañeros de trabajo, amigos, vecinos, etcétera), los denominados sistemas de apoyo naturales (o informales), así como también por las instituciones sanitarias y sus profesionales (médicos, personal de enfermería, etcétera), designados como sistemas de apoyo organizados (o formales). ${ }^{26}$

\section{DISCUSIÓN}

Presentada al pie de cada tema, dentro de los hallazgos y de manera intercalada, por hallazgo destacado.

\section{CONCLUSIONES}

El enfoque fenomenológico interpretativo proporciona a los profesionales de la salud un acercamiento con el paciente a partir de su propia subjetividad, la cual implica una serie de relaciones e interacciones que influyen en su condición de salud. Parte de estas interacciones resaltan los aspectos psicosociales que se generan a través de la esfera psicológica y social de la persona, lo que produce un resultado positivo o negativo en su propio autocuidado ante el padecimiento de la diabetes.

El lado psicosocial tiene una estrecha relación en el proceso de padecer una ENT, puesto que engloba aspectos relevantes que se reflejan en el cotidiano del paciente, debido a que existen cambios en el estilo de vida y en las relaciones interpersonales. Derivado de esto, se encontraron dos principales aspectos conductuales en las persona que padecen diabetes: el primero, caracterizado por la negación de la enfermedad, desinterés en los cuidados, falta de apego terapéutico, temor a la crítica social y deterioro de la autoestima (esto unido al duelo que se presenta por la pérdida de salud y un nuevo modo de vida); y el segundo, donde el paciente comprende su nuevo modo de vida a partir de la aceptación de la enfermedad y el apoyo recibido de sus redes sociales cercanas: la familia, amigos y compañeros de trabajo. En cuanto a su manera de sentir y pensar, manifiestan un estado intermitente de bienestar, ocasionando cambios en la autoestima y la motivación.

Es por todo lo anterior que los aspectos psicosociales se encuentran íntimamente relacionados con el proceso de salud y bienestar, los cuales son determinantes en la vida del individuo. Esto desde el propio diagnóstico de diabetes, el cual implica un forzoso cambio conductual y apego terapéutico de cara a las diversas complicaciones potenciales en las distintas esferas del paciente. Razón por la cual es competencia de los profesionales de la salud entender dichos aspectos de la diabetes desde la mirada subjetiva de quien la padece, para poder abonar a su aceptación y comprensión, guiándoles hacia un trayecto menos sinuoso y a una mejor calidad de vida.

\section{BiblografíA}

1. OMS. Enfermedades no transmisibles. [Sitio de Internet]. [Consultado: 29/12/2019]. Disponible en: http://bit.ly/1oYZqiU.

2. OMS. Informe mundial sobre la diabetes: resumen de orientación. [Sitio de Internet]. [Consultado: 29/12/2019]. Disponible en: https://bit.ly/3aJwjfj.

3. Ramos-Pérez V, Hernández-Pozo MR, Álvarez-Gasca MA. El afecto emocional en pacientes diabéticos mexicanos. Rev Tesis Psicológica. [Internet] 2019 [Consultado: 05/01/2020]; 14 (1): 1-21. Disponible en: https://bit.ly/39FvhAR.

4. Vinaccia S, Orozco LM. Aspectos psicosociales asociados con la calidad de vida de personas con enfermedades crónicas. Diversitas. [Internet] 2005 [Consultado: 05/01/2020]; 1 (2): 125-137. Disponible en: https://bit.ly/3cKuTTE.

5. Ledón L. Impacto psicosocial de la diabetes mellitus, experiencias, significadosy respuestas a la enfermedad. Rev Cubana Endocrinol [Internet] 2012 [consultado: 12/01/2020 ]; 23(1): 76-97. Disponible en: https://bit.ly/3gLIRqO

6. Soler SYM, Pérez RE, López SMC, Quezada RD. Conocimientos y autocuidado en pacientes con diabetes mellitus tipo 2. Rev Arch Med Camagüey. [Internet] 2016 [Consultado: 12/01/2020]; 20 (3): 244-252. Disponible en: https://bit.ly/3aKalDt.

7. García M. Comunicación y relaciones interpersonales. Tendencias pedagógicas. [Internet] 2015 [Consultado: 19/01/2020]; (2): 1-17. Disponible en: https://bit.ly/39gw2iR.

8. Álvarez-Gayou JL. Cómo hacer investigación cualitativa. Fundamentos y metodología. México: Paidós; 2003. p. 187-193.

9. Morse J. The significance of saturation. Qual Health Res. [Internet] 1995 [Consultado: 19/01/2020]; 5 (2): 147-149. Available in: https://bit.ly/39lj2DB. 
10. Morse J. Designing qualitative research. En: Denzin NK, Lincoln YS. Handbook of qualitative inquiry. Thousand Oaks: Sage publications; 1994. p. 220-235.

11. Emanuel E. ¿Qué hace que la investigación clínica sea ética? Siete requisitos éticos. En: Pellegrini-Filho A, Macklin R. Investigación en sujetos humanos: Experiencia Internacional. Programa Regional de Bioética. División de Salud y Desarrollo Humano. Organización Panamericana de la Salud. Serie Publicaciones; 1999, p. 43-44.

12. Ley General de Salud. Título quinto: Investigación para la Salud. México: Diario Oficial de la Federación, 3 febrero 1983. [Consultado: 26/01/2020]. Disponible en: https://bit. Iy/2IC5yNZ.

13. Castillo E, Vásquez ML. El rigor metodológico en la investigación cualitativa. Colomb Med. [Internet] 2003 [Consultado: 26/01/2020]; 3 (4): 164-167. Disponible en: https://bit.ly/336E3Fy.

14. Arias MM, Giraldo CV. El rigor científico en la investigación cualitativa. Invest Educ Enferm. [Internet] 2011 [Consultado: 02/02/2020]; 29 (3): 500-514. Disponible en: https://bit. $\mathrm{ly} / 38 \mathrm{GzFlt}$.

15. Guerrero-Castañeda RF, Menezes TM, Prado ML. La fenomenología en investigación de enfermería: reflexión en la hermenéutica de Heidegger. Es Anna Nery [Internet]. 2019 [consultado: 19/02/2020]; 23(4): 1-7. https://doi. org/10.1590/2177-9465-ean-2019-0059.

16. Chóliz M. Psicología de la emoción: el proceso emocional. Universidad de Valencia: España, 2005 [Consultado: 23/02/2020]. Disponible en: https://bit.ly/33b7pmm.

17. Páez D, Zuebieta EM, Mayordomo S, Jiménez A, Ruiz S. Identidad. Autoconcepto, autoestima, autoeficacia y locus de control. Psicologís social, cultura y educación. [Internet] 2005 [consultado: 24/02/2020]; p. 125.194. Disponible en: https://bit.ly/3ejcEnP

18. Centro Regional de Investigaciones Multidisciplinarias. Representaciones sociales y contextos de investigación con perspectiva de género. México: UNAM, 2013 [Consultado: 23/02/2020]. Disponible en: https://bit.ly/2xs5gal.
19. Castillo M, Marín L, Almenares K. Adherencia terapéutica y factores influyentes en pacientes con diabetes mellitus tipo 2. Rev Cubana Med Gen Integr. [Internet] 2017 [Consultado:24/02/2020]; 33 (4):1-10. Disponible en: https:// bit.ly/2WNwSGi.

20. Ávalos CM, López RC, Morales GM, Priego AH, Garrido PS, Cargill FN. Calidad en el control de la diabetes mellitus en unidades de atención primaria de México. Un estudio desde la perspectiva de la familia de los pacientes. Atención Primaria. [Internet] 2017 [Consultado:24/02/2020]; 49 (1): 2127. http://dx.doi.org/10.1016/j.aprim.2016.02.012.

21. Guerrero-Pacheco R, Galán-Cuevas S, Sánchez-Armáss O. Factores sociodemográficos y psicológicos asociados al autocuidado y la calidad de vida en adultos mexicanos con diabetes Mellitus tipo 2. Acta Colombiana de Psicología. [Internet] 2017 [Consultado: 12/01/2020]; 20 (2): 158-167. http://www.dx.doi.org/10.14718/ACP.2017.20.2.8.

22. Martínez B, Torres LE. Importancia de la familia en el paciente con diabetes mellitus insulinodependiente. Psicología y Salud. [Internet] 2007 [Consultado:25/02/2020]; 17 (2): 229-241. Disponible en: https://bit.ly/2IJCSD3.

23. Salazar JC, Guerrero JC, Machado YB, Cañedo R. Clima y cultura organizacional: dos componentes esenciales en la productividad laboral. ACIMED. [Internet] 2009 [Consultado: 26/02/2020]; 20 (4): 67-75. Disponible en: https://bit.ly/38JDQt4.

24. Cohen S, Gottlieb BH, Underwood LG. Social relationships and health. En Cohen S, Underwood LG, Gottlieb B. H. Social support measurement and intervention: A guide for health and social scientists. EUA: Oxford University Press.

25. Hernández S, Pozo C, Alonso E, Pérez E. Apoyo social y adherencia al tratamiento en pacientes crónicos. En: Romay J, García R, Eulogio J. Psicología social y problemas sociales. España: Biblioteca Nueva; Vol. 4. Psicología de la salud. 2005. p. 505-212.

26. Pineda M, Ortega RM, Carberí R. Sobre crónicos, cuidadores y sus familias. La necesidad de un nuevo planteamiento. Valencia: España: Consellería de Sanitat; 2001. p. 51. 